\title{
Crossover behavior of conductivity in a discontinuous percolation model
}

\author{
Seongmin Kim, ${ }^{1}$ Y.S. Cho, ${ }^{1}$ N.A.M. Araújo, ${ }^{2}$ and B. Kahng ${ }^{1}$ \\ ${ }^{1}$ Department of Physics and Astronomy, Seoul National University 151-747, Korea \\ ${ }^{2}$ Computational Physics for Engineering Materials, IfB, \\ ETH Zurich, Wolfgang-Pauli-Strasse 7, CH-8093 Zurich, Switzerland
}

\begin{abstract}
When conducting bonds are occupied randomly in a two-dimensional square lattice, the conductivity of the system increases continuously as the density of those conducting bonds exceeds the percolation threshold. Such a behavior is well known in percolation theory; however, the conductivity behavior has not been studied yet when the percolation transition is discontinuous. Here we investigate the conductivity behavior through a discontinuous percolation model evolving under a suppressive external bias. Using effective medium theory, we analytically calculate the conductivity behavior as a function of the density of conducting bonds. The conductivity function exhibits a crossover behavior from a drastically to a smoothly increasing function beyond the percolation threshold in the thermodynamic limit. The analytic expression fits well our simulation data.

PACS numbers: 64.60.ah,02.50.Ey,89.75.Hc
\end{abstract}

The concept of percolation transition has played a central role as a model for the formation of a spanning cluster connecting two opposite edges of a system in Euclidean space as a control parameter $p$ is increased beyond a certain threshold $p_{c}$ [1]. This model has been used to study many phenomena such as metal-insulator transitions and sol-gel transitions. The order parameter $P_{\infty}$ of percolation transition is defined as the probability that a bond belongs to a spanning cluster, which increases in the form $P_{\infty}(p) \sim\left(p-p_{c}\right)^{\beta}$ beyond $p_{c}$, where $p$ is a control parameter indicating the fraction of occupied bonds and $\beta$ is the critical exponent related to the order parameter. As an application of percolation model, one can construct a random resistor network in which each occupied bond is regarded as a resistor with unit resistance, and the system is in contact with two bus bars at the opposite edges of the system. When a voltage difference is applied between these two bus bars, the system is in a insulating state for $p<p_{c}$, but changes to conducting state for $p>p_{c}$, due to the formation of several conducting paths at $p_{c}$. Above $p_{c}$, the conductivity increases continuously as $g \sim\left(p-p_{c}\right)^{\mu}$, where $\mu$ is the conductivity exponent [2].

Recently the subject of discontinuous percolation transition (DPT) has been a central issue [3 12] with, for example, applicability to cascading failures in complex networks 13]. Among others 14 19], a model called spanning cluster avoiding (SCA) was introduced 20] aiming to generate a DPT. The DPT of the SCA model is rather trivial, for the percolation threshold is placed at $p_{c}=1$ in the thermodynamic limit, but for finite-sized systems $p_{c}<1$. Here, we study the conductivity as a function of $p$ in finite-sized systems for the SCA model. Indeed, we find that the conductivity increases drastically just after the percolation threshold and then exhibits a crossover to a smoothly increasing behavior. Such crossover has never been reported, though it is meaningful, as, a drastic change of conductivity in random resistor networks can find application, for example, on resistance switching phenomena in non-volatile memory devices [21]. From a theoretical perspective, the understanding of conductivity becomes complementary to the result on the percolation transition for the SCA model.

We first recall the SCA model. In this model, we take a two-dimensional regular square lattice of linear size $L$. Initially, the system consists of $N=L^{2}$ nodes and $2 N$ unoccupied bonds. At each time step, one randomly chooses $m$ unoccupied bonds, and those potential bonds are classified into two types: bridge and non-bridge bonds. Bridge bonds are those that would form a spanning cluster if any of them is occupied [22, 23]. One takes a non-bridge bond randomly among those $m$ candidates if exists. This choice suppresses the formation of a spanning cluster. As the number of occupied bonds is increased, the total number of bridge bonds $N_{B B}(p)$ increases and thus the probability that those $m$ bonds are all bridge bonds is also increased. If such a case happens, a bridge bond is inevitably occupied and a spanning cluster is formed. Once a spanning cluster is formed, no more restrictions are imposed on the occupation of bonds. It was found that when $m$ is greater than a tricritical point $m_{c} \approx 2.55$ in two dimensions, the percolation transition is discontinuous and the percolation threshold $p_{c m}$ approaches unity as the system size is increased [20]. In finite-sized systems, the percolation threshold $p_{c m}$ depends on the number of candidate bonds $m$. In this brief report, we present an analytic formula for the conductivity based on effective medium theory 24]. The analytic prediction of the conductivity function is in agreement with our numerical data.

In the SCA model, the percolation threshold is delayed by suppressing the formation of a spanning cluster. While the percolation threshold is delayed, two large clusters form independently, which are separated by bridge bonds. Bridge bonds form a fractal set of fractal dimension $d_{\mathrm{BB}} \approx 1.215[22]$. Moreover, for $m>m_{c}$, in those two separated clusters, the density of occupied bonds is extremely high, for $p$ close to and above $p_{c m}$. These facts enable us to apply effective medium theory to calculate the conductivity function near the percolation threshold 
(a)

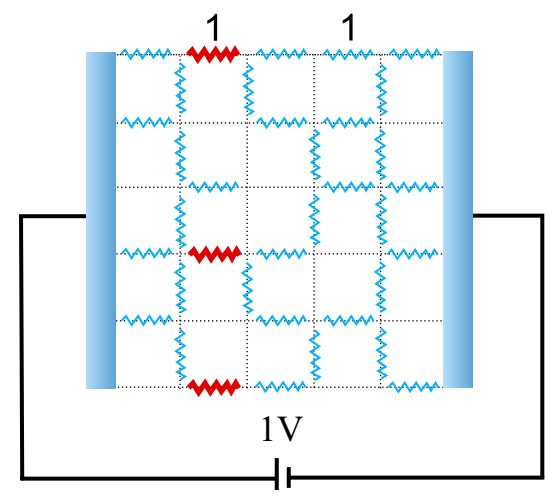

(b)

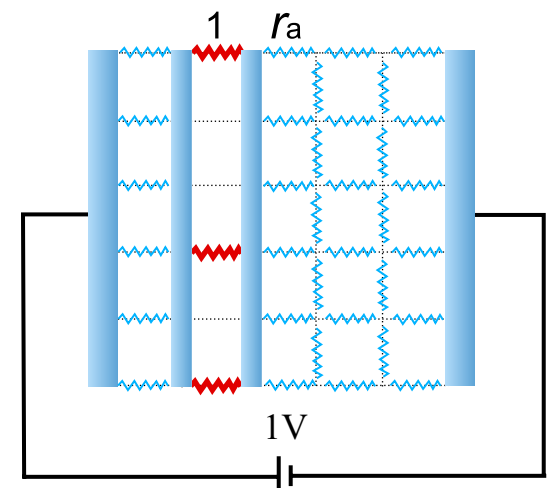

(c)

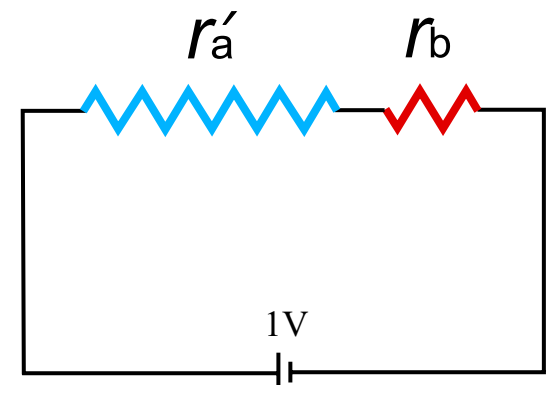

FIG. 1. (Color online) (a) Schematic diagram of circuit structure for $p \geq p_{c m}$ for the SCA model that consists of bonds of unit resistance. The occupied bonds are classified into original bridge bonds (thick red resistors) and original non-bridge bonds (thin blue resistors). (b) We simplify the whole circuit as series connection of a bundle of original bridge bonds of unit resistance and two compact clusters consist of bonds of resistance $r_{a}$ by applying effective medium theory. (c) The combined resistance of two compact clusters is calculated as $r_{a}^{\prime}=r_{a}(L-1) / L \approx r_{a}$ for large $L$, and the combined resistance of original bridge bonds $r_{b}$ is calculated as $r_{b}=1 / L p_{b}$, where the derivations are shown in the main text. We can calculate the conductivity as $g_{m}(p)=1 /\left(r_{a}^{\prime}+r_{b}\right)$.

for the SCA model. We recall the conductivity function for ordinary percolation obtained from effective medium theory near $p=1$, which is $g_{\mathrm{eff}}=2 p-1$ in two dimensions [24].

Next, we derive a formula for the conductivity using heuristic arguments. To proceed, we examine the structure of the system at the onset of the percolation transi-

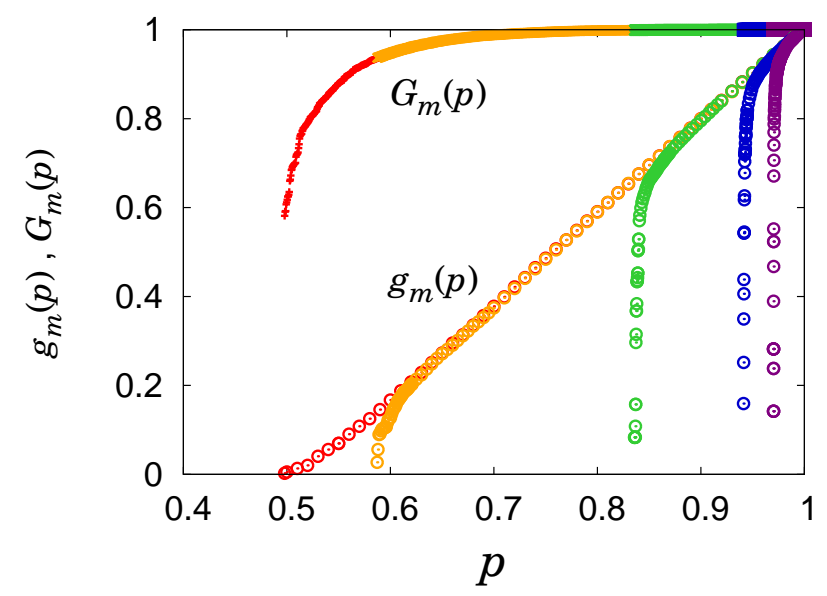

FIG. 2. (Color online) Plot of $G_{m}(p)$ and $g_{m}(p)$ vs $p . G_{m}(p)$ is the fraction of nodes belonging to the spanning cluster. $G_{m}(p)$ jumps to $G_{1}(p)$ from 0 at $p_{c m}$ and follows the envelop of $G_{1}(p)$ after that. $g_{m}(p)$ is the conductivity, it becomes positive at $p_{c m}$ and grows drastically after that. As $m$ is increased, $p_{c m}$ is delayed. Data are shown for $m=1$ (red), $m=2$ (yellow), $m=3$ (green), $m=4$ (blue), and $m=5$ (purple) from left to right. $L=300$ are considered. Results are for a single sample. tion, denoted as $p_{c m}^{-}$. As shown in Fig. 1(a), the system consists of two disconnected clusters separated by unoccupied bridge bonds. Due to the unoccupied separatrix, the conductivity of the system is zero at $p_{c m}^{-}$, but becomes nonzero once a bond among those bridge bonds is occupied as shown in the Fig. 2. Since the number of bridge bonds increases as $p$ increases for $p<p_{c m}$, from now on, we use the phrase "original bridge bonds" to refer to those bonds that were bridge bonds at $p_{c m}^{-}$. Similarly, "original non-bridge bonds" are all the other bonds. The densities of occupied bonds of original non-bridge bonds and original bridge bonds are denoted as $p_{a}$ and $p_{b}$, respectively. Those two densities depend on $p$, and $p_{b}=0$ at $p_{c m}^{-}$. Then, the following relation holds,

$$
2 L^{2} p \approx p_{a}\left(2 L^{2}-L^{d_{\mathrm{BB}}}\right)+p_{b} L^{d_{\mathrm{BB}}} \approx 2 L^{2} p_{a}+p_{b} L^{d_{\mathrm{BB}}},
$$

where we use the number of bridge bonds $N_{B B}\left(p_{c m}^{-}\right) \approx$ $L^{d_{B B}}$ and $L^{2} \gg L^{d_{\mathrm{BB}}}$ for large $L$. Then $p_{a} \approx p+$ $\mathcal{O}\left(1 / L^{2-d_{\mathrm{BB}}}\right)$. For $p_{b}(p)$, we use the fact that the occupation of original bridge bonds increases linearly with increasing $p$ for $p>p_{c m}$ and $p_{b}\left(p_{c m}\right)=0$. Then, one obtains,

$$
p_{b}(p)=\frac{p-p_{c m}}{1-p_{c m}} .
$$

In the spirit of effective medium theory, we assume that original non-bridge bonds are fully occupied but we consider that each bond has resistance $r_{a} \neq 1$. Next, we make a more crude assumption. Due to the fractal nature of the set of bridge bonds, the separatrix is not linear in its shape, and the density of original nonbridge and original bridge bonds are different. Thereby, the current can flow along the boundary between the 
original non-bridge and original bridge bonds. However, this current contribution to the conductivity of the system can be negligible when the system size is sufficiently large. Based on such facts, we simplify the system as shown in Figs. 1 (b) and (c). That is, the system consists of two parts, a rectangular-shape regular lattice of size $(L-1) \times L$ in which original all non-bridge bonds are all occupied with resistance $r_{a}=2 p_{a}-1$, and one dimensional columnar lattice of size $L$ in which original bridge bonds are occupied with probability $p_{b}$ and unit resistance. We also assume that there exists a busbar between the two parts, and thereby there is no net current on each vertical bond.

This simplified picture enables us to calculate the overall conductivity. The resistivity (the inverse of conductivity) is obtained as

$$
\frac{1}{g_{m}(p)} \approx \frac{1}{2 p_{a}-1}+\frac{1}{L p_{b}}
$$

where $g_{m}(p)$ denotes the conductivity at $p$ of the SCA model with the control parameter $m$. We compare the analytic result with our simulation data for different $m=2,3,4$, and 5. As can be seen in Fig. 3, the data for $L \times L=300 \times 300$ is in good agreement with the analytic expression for $m \geq 3$. Since for these cases $p_{c m}$ is close to unity (for example, $p_{c 3} \approx 0.84, p_{c 4} \approx 0.94$, and $p_{c 5} \approx 0.97$ ), the approximation based on effective medium theory is more accurate. For $m=2$, the data clearly differs from the analytic expression. Actually, the percolation threshold for $m=2$ reduces to the one of the ordinary percolation in the thermodynamic limit and, therefore, the two clusters connected through original bridge bonds cannot be considered compact, as necessary to apply effective medium theory.

Finally, we recall the previous result [20] that $p_{c m}$ approaches to one as $L$ is increased as

$$
1-p_{c m} \sim L^{-\frac{2}{m-1}\left(\frac{m}{m_{c}}-1\right)} \text { for } m>m_{c}
$$

where $m_{c} \approx 2.55$ in two dimensions. Then, $L p_{b} \equiv L^{\alpha}(p-$ $\left.p_{c m}\right)$, where

$$
\alpha=1+\frac{2}{m-1}\left(\frac{m}{m_{c}}-1\right) .
$$

Numerically, $\alpha \approx 1.18,1.38$, and 1.48 for $m=3$, 4 , and 5 in two dimensions, respectively. Depending on the magnitude of $p-p_{c m}$, the conductivity behaves as follows,

$$
g_{m}(p) \approx \begin{cases}L^{\alpha}\left(p-p_{c m}\right) & \text { for } \delta \ll 1 / L^{\alpha} \\ 2 p-1 & \text { for } \delta \gg 1 / L^{\alpha}\end{cases}
$$

where $\delta=p-p_{c m}$. Thus, there exists a crossover in the conductivity for $\delta_{c} \approx 1 / L^{\alpha}$. We remark that the conductivity increases more rapidly to $2 p_{c m}-1$ for larger systems due to the prefactor $L^{\alpha}$.

In summary, we studied the conductivity transition of two dimensional SCA model. In this model, $p_{c m}$ increases to 1 for $m>m_{c} \approx 2.55$, but otherwise it decreases to $p_{c 1}=0.5$ as the system size increases. We used effective medium theory which is valid for $p \gg p_{c 1}$ to calculate the analytic expression of conductivity in this model. We numerically confirmed the validity of this expression for $m=2,3,4$, and 5 in finite sized systems and found that the data is well fitted for $m=3,4$, and 5 . However, the case $m=2$ cannot be described by our theory.

This work was supported by the NRF grants (Grant No.2010-0015066) and the Global Frontier Program (YSC).
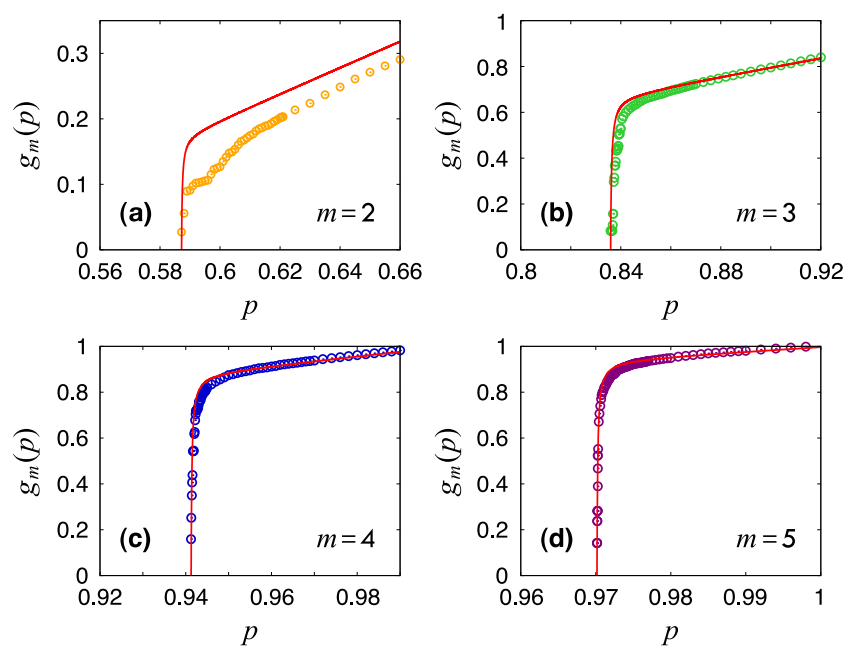

FIG. 3. (Color online) Plot of $g_{m}(p)$ vs $p$ for one sample with $L=300$. Just after $p_{c m}, g_{m}(p)$ becomes positive and grows drastically. Red solid lines are obtained from Eq. (3) for $m=2$ (a), $m=3$ (b), $m=4$ (c), and $m=5$ (d). We can find that the theoretical formula fits well the simulation data when $m$ is larger than the tricritical point $m_{c} \approx 2.55$.
[1] D. Stauffer and A. Aharony, Introduction to percolation theory, 2nd Ed. (Taylor \& Francis Inc, Philadelphia, USA 1991).

[2] N. Posé, N. A. M. Araújo, and H. J. Herrmann, Phys. Rev. E 86, 051140 (2012).

[3] D. Achlioptas, R. M. D'Souza, and J. Spencer, Science 323, 1453 (2009).
[4] R. M. Ziff, Phys. Rev. Lett. 103, 045701 (2009).

[5] A. A. Moreira, E. A. Oliveira, S. D. S. Reis, H. J. Herrmann, and J. S. Andrade, Jr., Phys. Rev. E 81, 040101(R) (2010).

[6] Y. S. Cho, J. S. Kim, J. Park, B. Kahng, and D. Kim, Phys. Rev. Lett. 103, 135702 (2009).

[7] Y. S. Cho, B. Kahng, and D. Kim, Phys. Rev. E 81, 
030103(R) (2010).

[8] Y.S. Cho and B. Kahng, Phys. Rev. E 84, 050102(R) (2011).

[9] N. A. M. Araújo, J. S. Andrade Jr., R. M. Ziff, and H. J. Herrmann, Phys. Rev. Lett. 106, 095703 (2011).

[10] J. S. Andrade, Jr., H. J. Herrmann, A. A. Moreira, and C. L. N. Oliveira, Phys. Rev. E 83, 031133 (2011).

[11] R. A. da Costa, S. N. Dorogovtsev, A. V. Goltsev, and J. F. F. Mendes, Phys. Rev. Lett. 105, 255701 (2010).

[12] O. Riordan and L. Warnke, Science 333, 322 (2011).

[13] S.V. Buldyrev, R. Parshani, G. Paul, H.E. Stanley, and S. Havlin, Nature 464, 1025 (2010).

[14] N. A. M. Araújo and H. J. Herrmann, Phys. Rev. Lett. 105, 035701 (2010).

[15] K. J. Schrenk, N. A. M. Araújo, and H. J. Herrmann, Phys. Rev. E 84, 041136 (2011).

[16] J. Nagler, A. Levina, and M. Timme, Nat. Phys. 7, 265
(2011).

[17] W. Chen and R. M. D'Souza, Phys. Rev. Lett. 106, 115701 (2011).

[18] K. J. Schrenk, A. Felder, S. Deflorin, N. A. M. Araújo, R. M. D'Souza, and H. J. Herrmann, Phys. Rev. E 85, 031103 (2012).

[19] Y.S. Cho and B. Kahng, Phys. Rev. Lett. 107, 275703 (2011).

[20] Y.S. Cho, S.M. Hwang, H.J. Herrmann, and B. Kahng, Science 339, 1185 (2013).

[21] S.C. Chae, et al., Adv. Mater. 20, 1154 (2008).

[22] K. J. Schrenk, N. A. M. Araújo, J. S. Andrade Jr., and H. J. Herrmann, Sci. Rep. 2, 348 (2012).

[23] E. Daryaei, N. A. M. Araújo, K. J. Schrenk, S. Rouhani, and H. J. Herrmann. Phys. Rev. Lett. 109, 218701 (2012).

[24] S. Kirkpatrick, Rev. Mod. Phys. 45, 574 (1973). 